\title{
Circ_000 I 944 Contributes to Glycolysis and Tumor Growth by Upregulating NFAT5 Through Acting as a Decoy for miR-142-5p in Non-Small Cell Lung Cancer
}

\author{
Yawei Dou' \\ Wei Tian' \\ Hongtao Wang' \\ Shanshan $\mathrm{Lv}^{2}$ \\ 'Department of Thoracic Surgery, \\ Shaanxi Province People's Hospital, Xi'an, \\ 7I0068, People's Republic of China; \\ ${ }^{2}$ Department of Cardiovascular Surgery, \\ Xijing Hospital of Airforce Medical \\ University, Xi'an, 710032, People's \\ Republic of China
}

Background: Circular RNAs (circRNAs) participate in the tumorigenesis of various cancers. CircRNA hsa_circ_0001944 (circ_0001944), derived from the TCONS_12_00030860 gene, has been uncovered to be upregulated in NSCLC (non-small cell lung cancer). Nevertheless, the influence of circ_0001944 on glycolysis and tumor growth in NSCLC is unclear.

Methods: Expression trend of circ_0001944 in NSCLC tissues and cells were evaluated by quantitative real-time polymerase chain reaction (qRT-PCR). Loss-of-function experiments were performed to assess the influence of circ_0001944 knockdown on proliferation, migration, invasion, and glycolysis of NSCLC cells. Protein levels were assessed by Western blotting. The regulatory mechanism of circ_0001944 was analyzed by bioinformatics analysis, dual-luciferase reporter assay, and/or RNA pull-down assay. The tumorigenicity of circ_0001944 was confirmed by xenograft assay.

Results: Circ_0001944 was highly expressed in NSCLC, and NSCLC patients with high expression of circ_0001944 had a worse prognosis. Circ_0001944 silencing decreased xenograft tumor growth in vivo and repressed proliferation, migration, invasion, and glycolysis of NSCLC cells in vitro. Circ_0001944 was verified as a decoy for microRNA (miR)142-5p, which targeted NFAT5 (nuclear factor of activated T cells 5). MiR-142-5p was downregulated while NFAT5 was upregulated in NSCLC. Both miR-142-5p inhibition and NFAT5 overexpression offset the suppressive impact of circ_0001944 silencing on proliferation, migration, invasion, and glycolysis of NSCLC cells. Circ_0001944 adsorbed miR-142$5 p$ to elevate NFAT5 expression in NSCLC cells.

Conclusion: Circ_0001944 promotes proliferation, migration, invasion, and glycolysis of NSCLC cells by upregulating NFAT5 through adsorbing miR-142-5p, offering a novel mechanism for understanding the advancement of NSCLC.

Keywords: NSCLC, circ_0001944, miR-142-5p, NFAT5, glycolysis

\section{Introduction}

Lung cancer is considered the number one killer in the world. Non-small cell lung cancer (NSCLC), which is a major subtype of lung cancer, is usually treated by surgery, molecular targeted therapy, immunotherapy, radiotherapy, and/or chemotherapy. ${ }^{1}$ For early NSCLC patients, their 5-year survival rate can reach about $56 \%$ after treatment. ${ }^{2}$ However, a large segment of NSCLC patients are
Correspondence: Shanshan Lv Department of Cardiovascular Surgery, Xijing Hospital of Airforce Medical University, No. 169, Changle West Road, Xi'an, 710032, People's Republic of China Tel +86-29-84775313

Email Ivshanshan202012@I63.com 
frequently diagnosed at an advanced stage and have a 5-year survival rate as low as about $4 \%{ }^{3,4}$ Hence, understanding the molecular mechanisms that accelerate the advancement of NSCLC is crucial for the development of effective treatment strategies.

Cancer cells, whose carbohydrate metabolism pattern is different from that of normal cells, mainly undergo glycolysis even when oxygen is sufficient. ${ }^{5}$ This metabolism of cancer cells is called the Warburg effect, which results in increased glucose uptake and lactate production, as well as reduced ATP production. ${ }^{6}$ Therefore, investigating glycolysis is essential for understanding the progression of NSCLC.

Circular RNAs (circRNAs), single-stranded RNAs with closed-loop structures, are formed by reverse splicing of premRNAs. ${ }^{7}$ In contrast to linear RNAs, they are resistant to RNase $\mathrm{R}$ and have a longer half-life. ${ }^{8}$ Also, circRNAs can function as a decoy and regulate gene expression through adsorbing microRNAs (miRs) via miR responsive elements. ${ }^{9}$ A large number of researches have unmasked that the anomalous expression of circRNAs is implicated in diverse human diseases, including cancer. $^{10}$ For instance, circRNA TMEM87A facilitated gastric cancer cell metastasis and proliferation through upregulating ULK1. ${ }^{11}$ CircRNA circ_0001320 adsorbed miR-558 to elevate TPM1 and TNFAIP1 expression, resulting in curbing cell invasion and growth in lung cancer. $^{12}$ CircRNA hsa_circ_0001944 (circ_0001944), located on chrX: 130883333-130928494, is derived from the TCONS_12_00030860 gene. Circ_0001944 had been uncovered to exert a promoting influence on the tumorigenesis of bladder cancer. ${ }^{13}$ Moreover, circ_0001944 was highly expressed in NSCLC tissues. ${ }^{14}$ At present, the role of circ_0001944 in NSCLC has not been verified.

Thus, we aimed to survey the effect of circ_0001944 on glycolysis and tumor growth, as well as its regulatory mechanism in NSCLC.

\section{Materials and Methods Clinical Samples}

47 NSCLC patients were recruited for the research and they signed informed consents. None of the recruited patients received any preoperative chemotherapy or radiotherapy. The study was approved by the Ethics Committee of the Shaanxi Province People's Hospital and conducted in accordance with the Declaration of Helsinki. All clinicopathological factors of NSCLC patients were exhibited in Table 1. Fresh NSCLC tissues and neighbor normal tissues $(>5 \mathrm{~cm}$
Table I Association of Clinicopathological Factors with circ_0001944 Expression in NSCLC Patients

\begin{tabular}{|c|c|c|c|c|}
\hline \multirow[t]{2}{*}{ Characteristics } & \multirow[t]{2}{*}{$\mathbf{n}$} & \multicolumn{2}{|c|}{ circ_0001944 Expression } & \multirow[t]{2}{*}{$\mathbf{P}$} \\
\hline & & High $(n=24)$ & Low $(n=23)$ & \\
\hline \multicolumn{5}{|l|}{ Age(years) } \\
\hline$\geq 60$ & 25 & 15 & 10 & 0.191 \\
\hline$<60$ & 22 & 9 & 13 & \\
\hline \multicolumn{5}{|l|}{ Gender } \\
\hline Male & 31 & 18 & 13 & 0.181 \\
\hline Female & 16 & 6 & 10 & \\
\hline \multicolumn{5}{|l|}{ Smoking history } \\
\hline Yes & 27 & 17 & 10 & 0.058 \\
\hline No & 20 & 7 & 13 & \\
\hline \multicolumn{5}{|l|}{ Tumor size $(\mathrm{cm})$} \\
\hline$\geq 4$ & 26 & 16 & 10 & 0.110 \\
\hline$<4$ & 21 & 8 & 13 & \\
\hline \multicolumn{5}{|l|}{ TNM stage } \\
\hline $1 / I 1$ & 28 & 10 & 18 & $0.011^{*}$ \\
\hline III & 19 & 14 & 5 & \\
\hline \multicolumn{5}{|l|}{ Lymphatic metastasis } \\
\hline Yes & 27 & 19 & 8 & $0.002^{*}$ \\
\hline No & 20 & 5 & 15 & \\
\hline \multicolumn{5}{|l|}{ Distant metastasis } \\
\hline Yes & 15 & 12 & 3 & $0.007^{*}$ \\
\hline No & 32 & 12 & 20 & \\
\hline
\end{tabular}

$* p<0.05$

away from the tumor) were obtained from 47 NSCLC patients who were diagnosed and received thoracic surgery at Shaanxi Province People's Hospital and then freshly frozen by liquid nitrogen and deposited at $-80^{\circ} \mathrm{C}$ fridge.

\section{Cell Culture}

Normal lung epithelial cells (BEAS-2B) and NSCLC cells (A549, H1975, H522, and HCC827) were obtained from BeNa Culture Collection (Beijing, China). All cells were maintained in a humidified chamber at $37^{\circ} \mathrm{C}$ with $5 \% \mathrm{CO}_{2}$. These NSCLC cell lines were cultured in RPMI (Roswell Park Memorial Institute)-1640 medium (Thermo Fisher, Waltham, MA, USA) supplemented with $10 \%$ FBS (fetal bovine serum) (Thermo Fisher) and 1\% P/S (penicillin/streptomycin) (Sigma, St. Louis, MO, USA), but BEAS-2B cells were cultured in basal BEGM (bronchial epithelial cell growth medium) (Lonza, Walkersville, MD, USA) supplemented with standardized growth factors (BEGM BulletKit, Lonza). 


\section{Stable Knockdown of circ_000I944 in NSCLC Cells}

To generate NSCLC cells (A549 and H1975) with stable knockdown of circ_0001944, a short hairpin (sh) RNA targeting circ_0001944 (sh-circ_0001944) and matching negative control (NC) (sh-NC) were synthesized by Sangon (Shanghai), followed by inserting into the pLKO.1 vector (Thermo Fisher), respectively. Subsequently, these produced vectors were transfected into HEK293T cells (BeNa Culture Collection) together with lentiviral packaging plasmids using the Lipofectamine 3000 reagent (Thermo Fisher), respectively. $72 \mathrm{~h}$ later, these lentiviral particles were collected and then used to infect NSCLC cells. These NSCLC cells were then cultured in the cell medium containing puromycin $(2 \mu \mathrm{g} / \mathrm{mL})$ (Sigma) to select NSCLC cells with a stable knockdown of circ_0001944.

\section{Oligonucleotides and Plasmids}

MiR-142-5p inhibitor and mimic (anti-miR-142-5p and miR-142-5p), as well as their matching NCs (anti-miR$\mathrm{NC}$ and miR-NC), were synthesized by Sangon (Shanghai). To generate the pcDNA-NFAT5 (NFAT5) plasmid, the full-length sequence of NFAT5 was inserting into the pcDNA vector (Thermo Fisher). Transient transfection was conducted using the Lipofectamine 3000 reagent (Thermo Fisher).

\section{Quantitative Real-Time Polymerase Chain Reaction (qRT-PCR)}

Total RNA was extracted using the Trizol reagent (Thermo Fisher). The complementary DNA was produced using the HiScript II 1st Strand Complementary DNA Synthesis Kit (Vazyme, Nanjing, China) or miScript II RT Kit (QIAGEN, Hilden, Germany). QRT-PCR was conducted using the ChamQ SYBR qPCR Master Mix (Vazyme). Relative expression was determined by the $2^{-\Delta \Delta \mathrm{Ct}}$ method. $\beta$-actin and U6 acted as endogenous controls. Each experiment was performed in triplicate. All primer sequences were synthesized by Sangon and presented in Table 2 .

\section{3-(4,5-Dimethylthiazol-2-yl)-}

\section{2,5-Diphenyltetrazolium Bromide (MTT) Assay}

Following transfection with oligonucleotides and/or plasmids, NSCLC cells $\left(1 \times 10^{3} /\right.$ well $)$ were seeded in 96-well plates (Thermo Fisher). After culturing for different time,
Table 2 Primer Sequences Used for qRT-PCR

\begin{tabular}{|c|c|}
\hline Genes & Primer Sequences $\left(5^{\prime}-3^{\prime}\right)$ \\
\hline \multirow[t]{2}{*}{ circ_0001944 } & Forward (F): 5'-TGCAGATACGATGCTGAGTGA-3' \\
\hline & $\begin{array}{l}\text { Reverse (R): 5'- } \\
\text { ACATACTGACACCTTAGTCTCCTC-3' }\end{array}$ \\
\hline \multirow[t]{2}{*}{ miR-142-5p } & F: 5'-CATAAAGTAGAAAGCACTAC-3' \\
\hline & R: 5'-GAACATGTCTGCGTATCTC-3' \\
\hline \multirow[t]{2}{*}{ miR-665 } & F: 5'-ACCAGGAGGCTGAGGC-3' \\
\hline & R: 5'-GAACATGTCTGCGTATCTC-3' \\
\hline \multirow[t]{2}{*}{ NFAT5 } & F: 5'-CCTAATGCCCTGATGACTCCAC-3' \\
\hline & R: 5'-GTTTGCTGAGTTGATCCAACAGAC-3' \\
\hline \multirow[t]{2}{*}{ PUM2 } & F: 5'-CGGTTAATGGCTCCAACACCTG-3' \\
\hline & R: 5'-CGAAACAGACCATTTGTGCTGCC-3' \\
\hline \multirow[t]{2}{*}{ TRIM36 } & F: 5'-ACCTGGTAAAAGTGGGAGTTGCT-3' \\
\hline & R: 5'-ACAGGCATCCTCACTTCCACTG-3' \\
\hline \multirow[t]{2}{*}{ ARMC8 } & F: 5'-GTGTAGAGCTGGAGCAATTCGG-3' \\
\hline & R: 5'-AGGCAAGTGTCTCAGCTCCTTC-3' \\
\hline \multirow[t]{2}{*}{$\mathrm{FBXO} 21$} & F: 5'-CCTTTCTTCAGCAGCCAGATGAC-3' \\
\hline & R: 5'-CAAACAAGCTCCACGATGCTGTC-3' \\
\hline \multirow[t]{2}{*}{ BTBD7 } & F: 5'-ACATGACCAGGGCAGAAGAAGC-3' \\
\hline & R: 5'-TGAAAGCTCTGCTGCACAGGCA-3' \\
\hline \multirow[t]{2}{*}{ U6 } & F: 5'-CTCGCTTCGGCAGCACA-3' \\
\hline & R: 5'-AACGCTTCACGAATTTGCGT-3' \\
\hline \multirow[t]{2}{*}{$\beta$-actin } & F: 5'-CTCGCCTTTGCCGATCC-3' \\
\hline & R: 5'-TCTCCATGTCGTCCCAGTTG-3' \\
\hline
\end{tabular}

$10 \mu \mathrm{L}$ MTT solution (Sigma) was added to each well, and the purple crystals were dissolved with $100 \mu \mathrm{L}$ dimethyl sulfoxide (Sigma). The OD (optical density) value (490 nm) was measured using a microplate detection system (SpectraMax i3x, Molecular Devices, San Jose, CA, USA).

\section{Colony Formation Assay}

Transfected NSCLC cells $\left(5 \times 10^{2} /\right.$ well $)$ were seeded in 6-well plates (Thermo Fisher). 10 days later, these colonies were stained with $0.1 \%$ crystal violet solution (Sigma), followed by calculating under a microscope 
(Nikon Eclipse E600, Nikon Instruments, Melville, NY, USA).

\section{Cell Migration and Invasion Assays}

Transwell chambers with different product numbers (\#3422 and \#354480, Costar, Cambridge, MA, USA) were used to evaluate the migration (\#3422) and invasion (\#354480) of transfected NSCLC cells. In brief, $200 \mu \mathrm{L}$ serum-free cell culture medium containing NSCLC cells $\left(1 \times 10^{5} /\right.$ chamber) was inoculated into the upper chambers. $600 \mu \mathrm{L}$ cell culture medium containing 10\% FBS (Thermo Fisher) was added to the lower chambers. 24 $\mathrm{h}$ later, the migrating and invading cells were stained with $0.1 \%$ crystal violet (Sigma), and the number of migrating and invading cells was calculated using a microscope (Nikon Eclipse E600, Nikon Instruments) under $\times 100$ amplification.

\section{Metabolic Analysis}

After culturing for $48 \mathrm{~h}$, glucose uptake and lactate production were measured using an automatic biochemical analyzer (7170A, HITACHI, Tokyo, Japan) as previously described. ${ }^{15}$ Relative glucose uptake rate and lactate production rate were normalized with the sample protein concentration.

\section{Western Blotting}

Total protein was extracted using the RIPA buffer (Thermo Fisher). $30 \mu \mathrm{g}$ total protein was separated by $12 \%$ sodium dodecyl sulfate-polyacrylamide gel electrophoresis and transferred onto polyvinylidene difluoride (PVDF) membranes (Bio-Rad, Hercules, CA, USA), followed by sealing with $5 \%$ non-fat milk. The membranes were incubated with primary antibodies against HK2 (Hexokinase II) (ab209847, 1:1000, Abcam, Cambridge, MA, USA), LDHA (Lactate Dehydrogenase A) (ab125683, 1:1000, Abcam), NFAT5 (ab3446, 1:1000, Abcam), and $\beta$-actin (ab179467, 1:5000, Abcam). The membranes were incubated with a secondary antibody, and the blots were detected by enhanced ECL substrate kit (Amersham, Piscataway, NJ, USA).

\section{Bioinformatics Analysis}

The miRs that might interact with circ_0001944 were predicted using Starbase and Circinteractome databases. The targets of miR-142-5p were predicted using miRDB, Starbase, Targetscan, microT, and miRWalk databases.

\section{Dual-Luciferase Reporter Assay}

In order to verify the putative binding sites, the luciferase reporters containing the sequence of circ_0001944-wt (wild type), circ_0001944-mut (mutant), NFAT5 3'UTR (untranslated region)-wt, and NFAT5 3'UTR-mut were constructed using the pMIR-REPORT reporter (Applied Biosystems, Foster City, CA, USA), respectively. NSCLC cells were transfected with miR-142-5p mimic or miR-NC together with a luciferase reporter carrying circ_0001944-wt, circ_0001944mut, NFAT5 3'UTR-wt, or NFAT5 3'UTR-mut, followed by evaluating the luciferase activity using a dual-luciferase reporter assay kit (BioVision, Milpitas, CA, USA).

\section{RNA Pull-Down Assay}

NSCLC cells were transfected with biotinylated (Bio)-miR -142-5p-wt, Bio-miR-142-5p-mut, or Bio-miR-NC (Sangon). The lysates of NSCLC cells were incubated with M-280 streptavidin beads (Sigma) that had been blocked with $10 \mu \mathrm{g} / \mathrm{mL}$ RNase-free BSA (bovine serum albumin) (Sigma) and yeast tRNA (Sigma). QRT-PCR was utilized to analyze the enrichment of circ_0001944.

\section{Xenograft Assay}

The animal experiments were approved by the Animal Ethics Committee of Shaanxi Province People's Hospital and conducted followed by Laboratory animal-Guideline for ethical review of animal welfare (GB/T 35892-2018). 12 male BALB/c nude mice (4-6 weeks old, 15-20 g) were obtained from Vital River Laboratory (Beijing, China) and divided into 2 groups by a random number table A549 cells with shcirc_0001944 or sh-NC were subcutaneously injected into the right flank of nude mice (every 6 mice in a group). Tumor volume was measured every 5 days with a digital caliper and calculated based on the following equation: Volume $=$ (length $\times$ width $\left.^{2}\right) / 2$. After injection for 30 days, all mice were euthanized under anesthesia to strip the tumor tissue for tumor weight evaluation and genetic analysis.

\section{Statistical Analysis}

Each experiment was performed in triplicate. Data were produced from three independent experiments and presented as mean \pm standard deviation. SPSS statistics 17.0 version (SPSS Inc., Chicago, IL, USA) was utilized for statistical analysis. Survival curves were plotted using the KaplanMeier method and compared by Log rank test. Unpaired and paired Student's $t$-test was used to compare between 2 groups. One-way analysis of variance with Tukey's test was 
used to compare three or more independent groups. Chisquare test was used to analyze the relationship between circ_0001944 expression and clinicopathological characteristics. Statistical significance was accepted when $P<0.05$.

\section{Results}

\section{NSCLC Patients with High circ_000I944 Expression Had a Poor Prognosis}

To validate the differential expression of circ 0001944 in NSCLC, we conducted qRT-PCR to detect circ_0001944 expression in 47 paired NSCLC tissues and neighboring normal tissues. As presented in Figure 1A, circ_0001944 had a higher level in NSCLC tissues than that in neighbor normal tissues. Also, circ_0001944 expression was associated with TNM stage, lymphatic metastasis, and distant metastasis in NSCLC patients (Table 1). Kaplan-Meier survival curves displayed a correlation between high circ_0001944 expression and poor prognosis of NSCLC patients (Figure 1B). As expected, circ_0001944 was observably upregulated in NSCLC cells (A549, H1975, H522, and HCC827) compared to the BEAS-2B cells, and circ_0001944 was higher expression in A549 and H1975 cells than that of other NSCLC cells, so A549 and H1975 cells were used for functional analysis (Figure 1C). Collectively, these results indicated that high circ_0001944 expression was associated with poor prognosis of NSCLC patients.

\section{Silencing of circ_0001944 Suppressed Proliferation, Migration, Invasion, and Decreased Glycolysis of NSCLC Cells}

Because circ_0001944 had a higher level in NSCLC cells, we constructed NSCLC cells with stable knockdown of circ_0001944 to survey the role of circ_0001944. The knockdown efficiency of sh-circ_0001944 in A549 and
H1975 cells is exhibited in Figure 2A. The silence of circ_0001944 repressed cell growth and cloned ability of A549 and H1975 cells (Figure 2B-E). Furthermore, circ_0001944 inhibition decreased migration and invasion abilities of A549 and H1975 cells (Figure 2F and G). We also explored the impact of circ_0001944 silencing on the glycolysis of NSCLC cells. The results exhibited that glucose uptake and lactate production in A549 and H1975 cells were significantly decreased after circ_0001944 inhibition (Figure 2H and I). As expected, the knockdown of circ_0001944 reduced protein levels of HK2 and LDHA in A549 and H1975 cells (Figure 2J and K). Together, these results manifested that circ_0001944 facilitated proliferation, migration, invasion, and elevated glycolysis of NSCLC cells.

\section{Circ_0001944 Acted as a Decoy for miR-I $42-5 p$}

To investigate the regulatory mechanism of circ_0001944 in NSCLC, we searched for miRs that might interact with circ_0001944 through bioinformatics analysis. As exhibited in Figure 3A, the seed sequences of miR-142-5p and miR665 were complementary to circ_0001944, which were jointly predicted in the Starbase and Circinteractome databases. Also, circ_0001944 knockdown elevated miR-142-5p expression in A549 and H1975 cells (Figure 3B and C). The putative binding sites of miR-142-5p on circ_0001944 are presented in Figure 3D. The expression of miR-142-5p was markedly increased in A549 and H1975 cells after miR-142$5 \mathrm{p}$ mimic transfection (Figure 3E). Furthermore, miR-142$5 \mathrm{p}$ mimic reduced the luciferase activity of the circ_0001944-wt reporter in A549 and H1975 cells but not the circ_0001944-mut reporter (Figure 3F and G). As expected, circ_0001944 could be pulled down by the BiomiR-142-5p-wt probe instead of Bio-miR-NC and Bio-miR $-142-5 p$-mut probes (Figure 3H). In addition, miR-142-5p
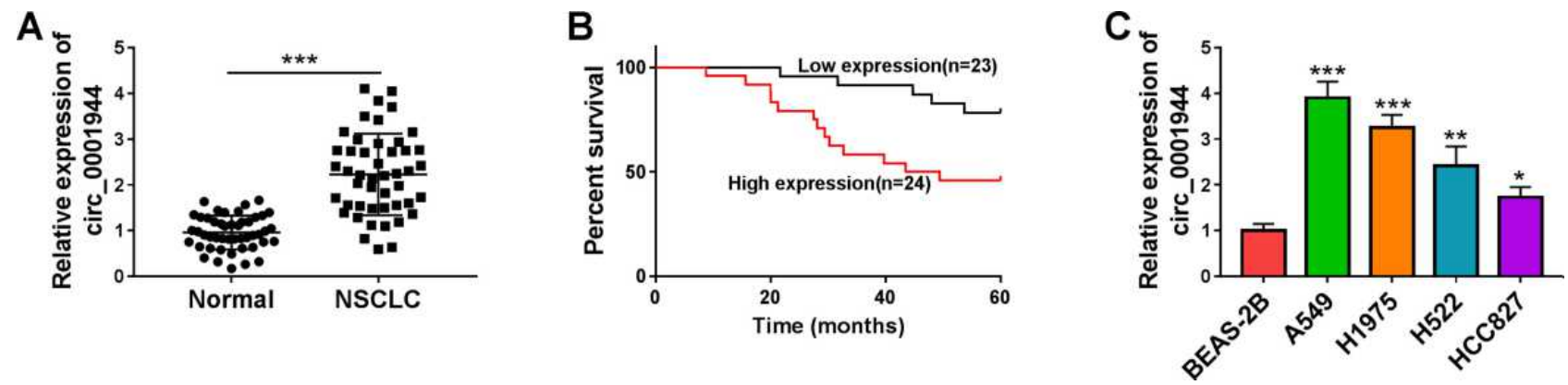

Figure I The prognosis of NSCLC patients with high expression of circ_0001944 was worse. (A) QRT-PCR analysis of circ_000I944 expression in 47 paired NSCLC tissues and neighbor normal tissues. (B) Kaplan-Meier survival curves showed the prognosis of NSCLC patients with high or low expression of circ_000I944. (C) Assessment of circ_0001944 expression in NSCLC cells (A549, HI975, H522, and HCC827) and the BEAS-2B cells by qRT-PCR. *P < 0.05 , **P < 0.01 , and ***P < 0.001 . 

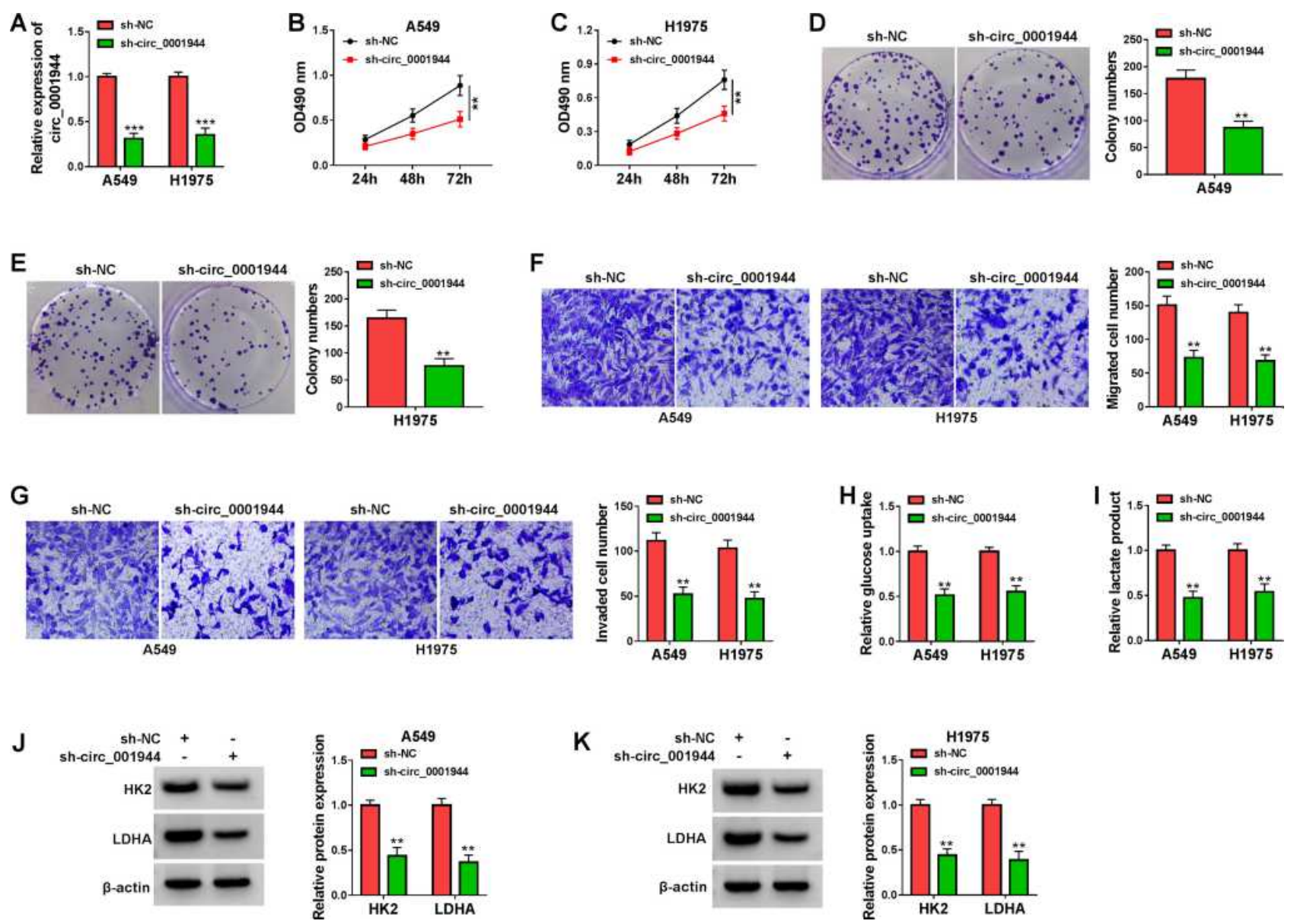

Figure 2 Circ_0001944 promoted proliferation, migration, invasion, and glycolysis of NSCLC cells. (A-K) A549 and HI975 cells were transfected with sh-circ_000I944 or sh-NC, and sh-NC was utilized as a control. (A) QRT-PCR validated the knockdown efficiency of sh-circ_000I944 in A549 and HI975 cells. (B-G) The proliferation, migration, and invasion of $\mathrm{A} 549$ and HI 975 cells were analyzed by MTT, colony formation, and transwell assays. (H and $\mathbf{I})$ Analysis of glucose uptake and lactate production in A549 and HI 975 cells using an automatic biochemical analyzer. ( $\mathbf{J}$ and $\mathbf{K}$ ) Western blotting detected protein levels of HK2 and LDHA in A549 and HI 975 cells. **P < 0.0 I and $* * * P<0.001$.

was lowly expressed in NSCLC tissues and was negatively correlated with circ_0001944 (Figure 3I and J). The expression of miR-142-5p was also decreased in NSCLC cells (A549 and H1975) (Figure 3K). In sum, these results suggested that circ_0001944 served as a sponge for miR-142-5p in NSCLC cells.

\section{Circ_0001944 Accelerated Malignancy and Glycolysis of NSCLC Cells by Sponging miR-I42-5p}

In view of the above findings, we further analyzed whether circ_0001944 sponged miR-142-5p to regulate malignancy and glycolysis of NSCLC cells. QRT-PCR exhibited that miR-142-5p expression in A549 and H1975 cells transfected with anti-miR-142-5p was decreased (Figure 4A). Moreover, the transfection of anti-miR-142-5p counteracted the suppressive influence of circ 0001944 silencing on proliferation, migration, and invasion of A549 and H1975 cells (Figure 4B-F). Also, the decrease in glucose uptake and lactate production in circ_0001944inhibited A549 and H1975 cells was reversed after miR142-5p silencing (Figure 4G and H). Additionally, miR142-5p inhibitor overturned the downregulation of HK2 and LDHA in A549 and H1975 cells caused by circ_0001944 knockdown (Figure 4I and J). These findings manifested that circ_0001944 contributed to malignancy and glycolysis of NSCLC cells by antagonizing miR-142-5p.

\section{NFAT5 Was a Target of miR-142-5p}

To survey the downstream targets of miR-142-5p in NSCLC, we sought for mRNAs that might be regulated by miR-142-5p through bioinformatics analysis. Venn analysis of miRDB, 
A

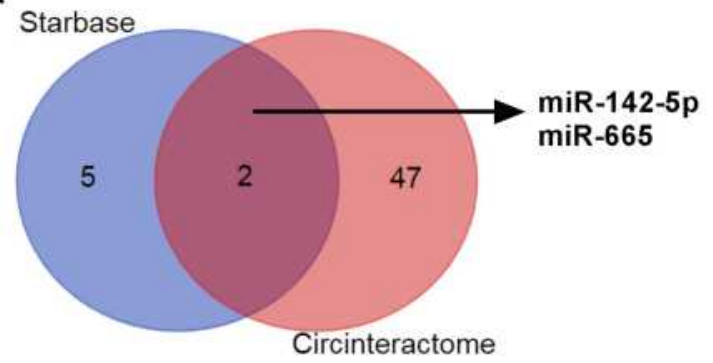

B

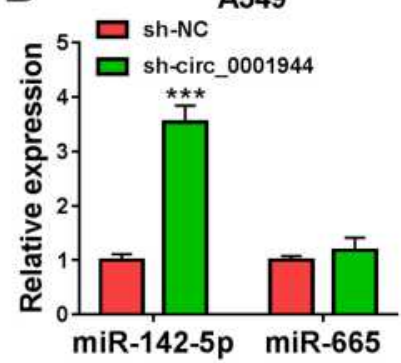

C

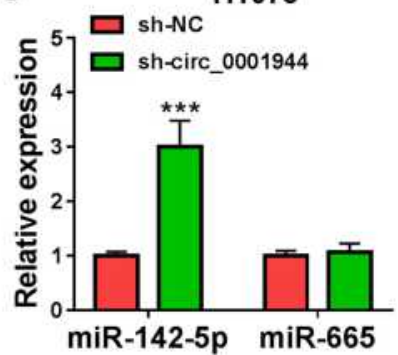

D

Hsa_circ_0001944 wt 5'-UUAAAGAGAGGAGAUACUUUAUG-3'

Hsa_miR-142-5p

3'-UCAUCACGAAAGAUGAAAUAC-5'

Hsa_circ_0001944 mut 5'-UUAAAGAGAGGAGAAUGAAAUAG-3'

$\mathbf{F}$
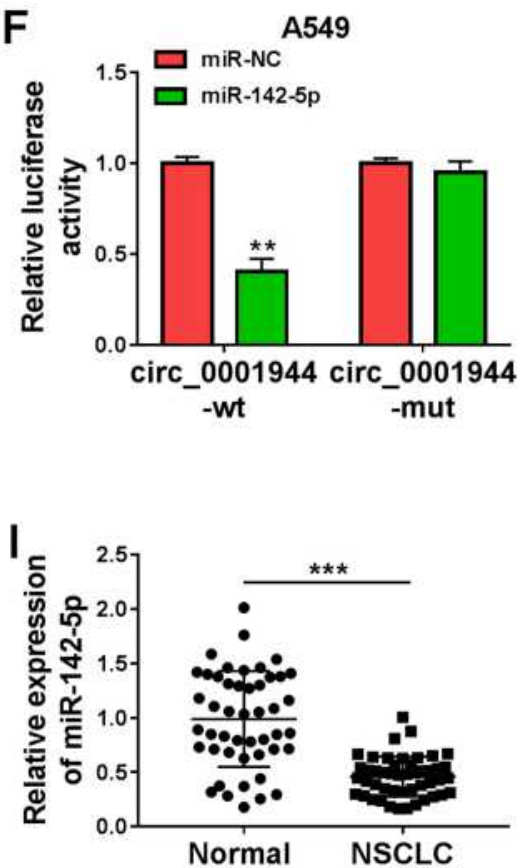

G
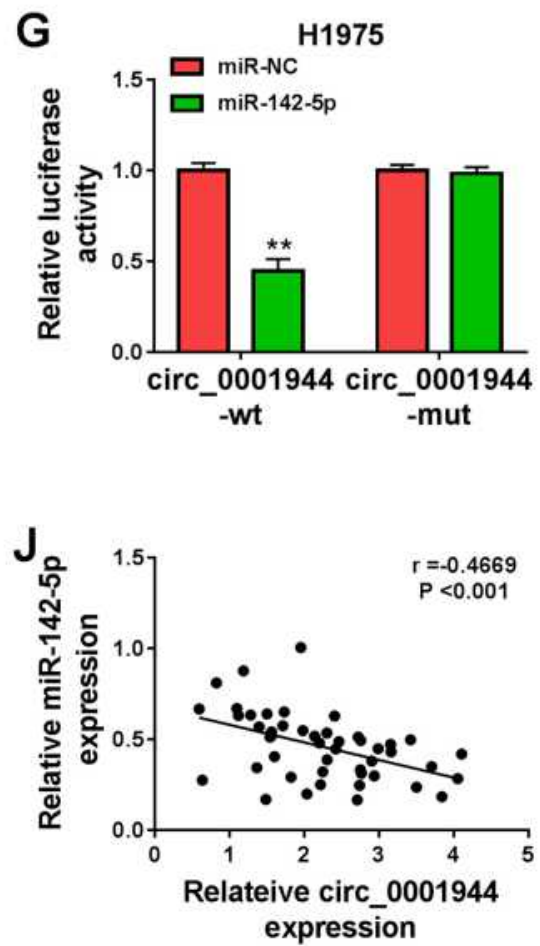
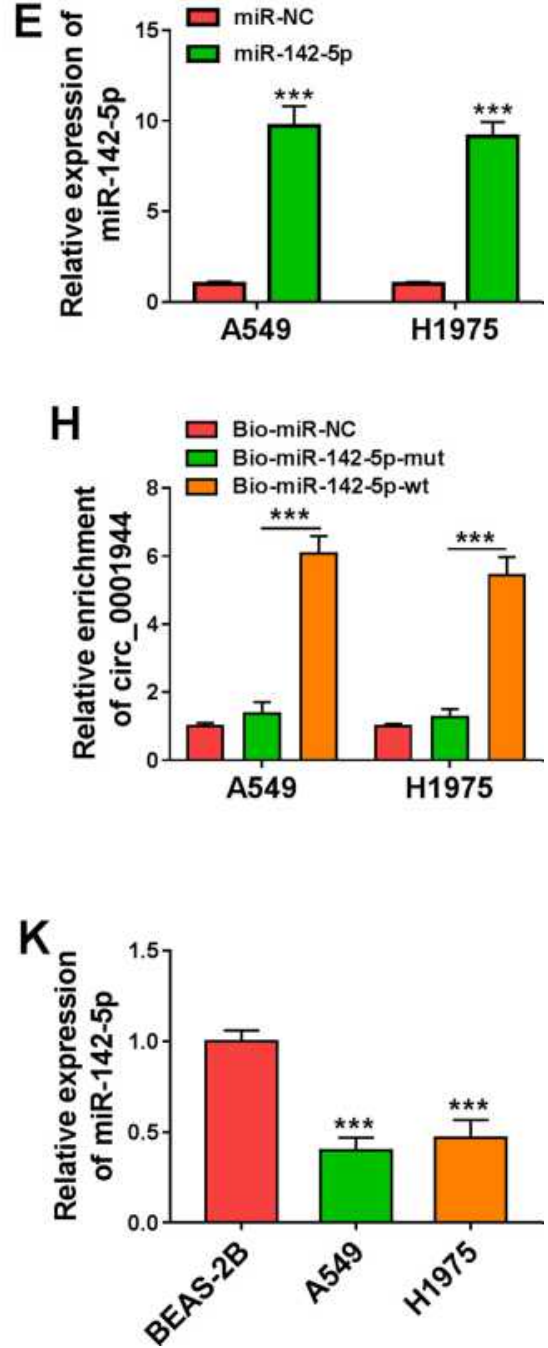

Figure 3 Circ_0001944 was verified as a sponge for miR-142-5p. (A) The schematic figure exhibited the overlapping miRs that might interact with circ_000I944 in the Starbase and Circinteractome databases. (B and C) QRT-PCR revealed the expression of miR-I42-5p and miR-665 in A549 and HI975 cells transfected with sh-circ _000I 944 or sh-NC. (D) The schematic figure presented the putative binding sites of miR-I42-5p on circ_000I944. (E) QRT-PCR detected the expression of miR-I42-5p in A549 and HI975 cells transfected with miR-142-5p mimic or miR-NC. (F and G) The putative binding sites between miR-I42-5p and circ_000I944 were verified by dualluciferase reporter assay. (H) QRT-PCR analyzed circ_000I944 expression in Bio-miR-142-5p-wt, Bio-miR-NC, and Bio-miR-142-5p-mut groups. (I) Expression pattern of miR-142-5p in NSCLC tissues was verified by qRT-PCR. (J) Analysis of the correlation between miR-142-5p and circ_000I944 expression in NSCLC tissues by Pearson's correlation analysis. (K) Expression trend of miR-142-5p in NSCLC cells was validated by qRT-PCR. $* * P<0.01$ and $* * * P<0.001$.

Starbase, Targetscan, microT, and miRWalk databases exhibited that there were 35 genes that might be targets of miR-1425p (Figure 5A). Next, we selected 6 genes (NFAT5, PUM2, TRIM36, ARMC8, FBXO21, and BTBD7) related to cancer for subsequent analysis. As displayed in Figure 5B and C, miR142-5p mimic only repressed the expression of NFAT5 mRNA in both A549 and H1975 cells. The potential binding sites of NFAT5 3'UTR on miR-142-5p are shown in Figure 5D. 
A
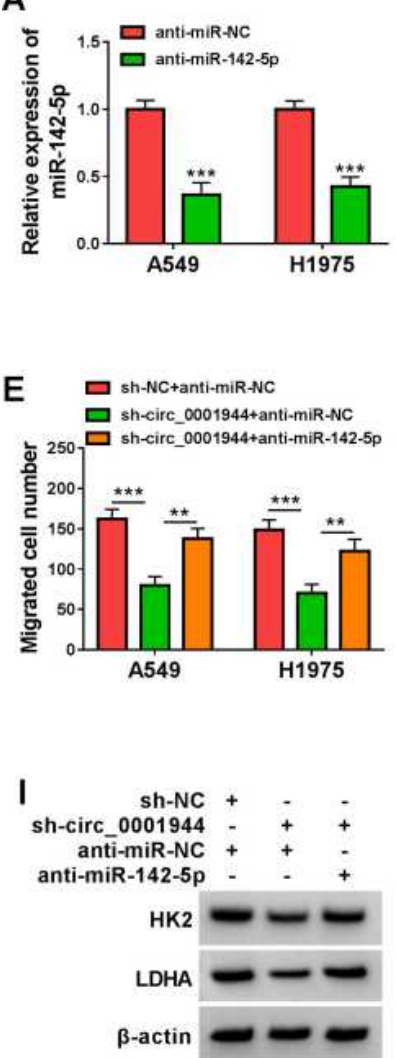
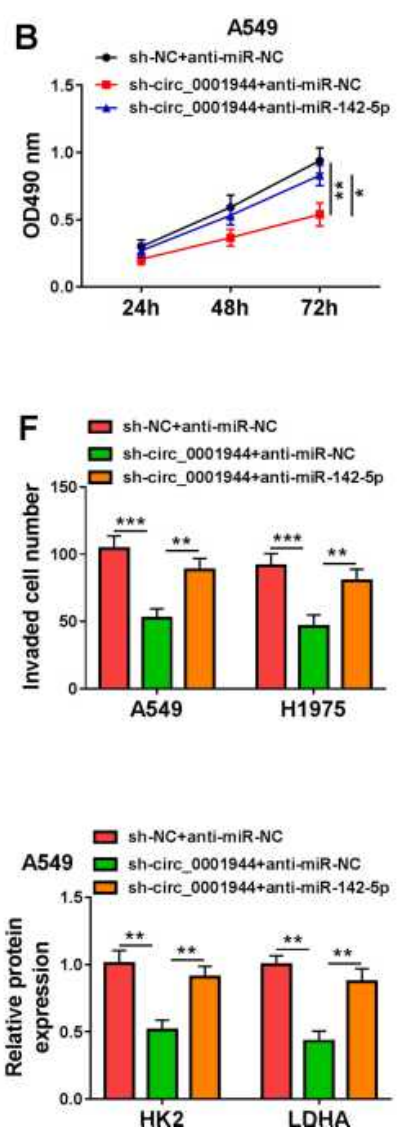
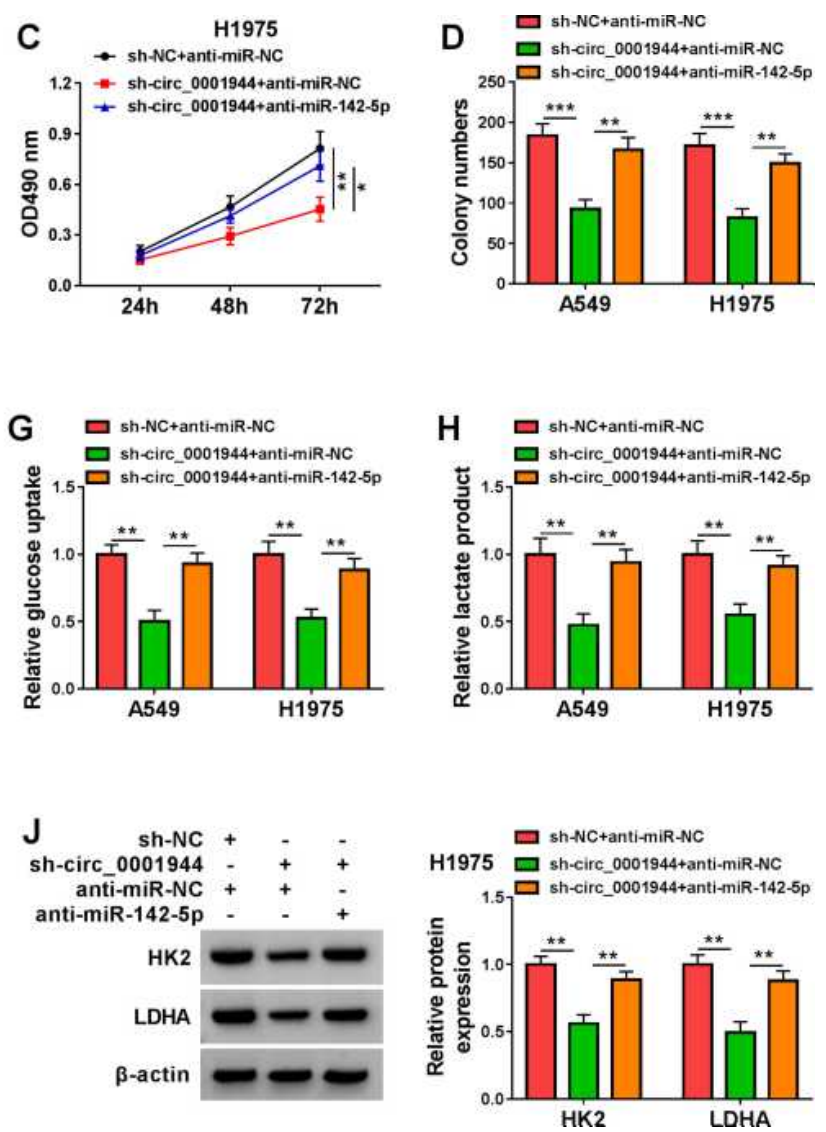

Figure 4 Circ_000I944 adsorbed miR-142-5p to regulate proliferation, migration, invasion, and glycolysis of NSCLC cells. (A) Expression level of miR-I42-5p in A549 and HI975 cells transfected with anti-miR-142-5p or anti-miR-NC was analyzed by qRT-PCR. (B-J) A549 and HI 975 cells were transfected with sh-NC+anti-miR-NC, sh-circ 0001944+anti-miR-NC, or sh-circ_0001944+anti-miR-I42-5p. (B-F) Analysis of proliferation, migration, and invasion of A549 and HI975 cells by MTT, colony formation, and transwell assays. ( $\mathbf{G}$ and $\mathbf{H}$ ) Evaluation of glucose uptake and lactate production in A549 and $\mathrm{H} 1975$ cells using an automatic biochemical analyzer. (I and J) Protein levels of HK2 and LDHA in A549 and HI975 cells were measured by Western blotting. $* P<0.05, * * P<0.01$, and $* * * P<0.001$.

Furthermore, the luciferase activity in A549 and H1975 cells co-transfected with miR-142-5p mimic and the NFAT5 3'UTR-wt reporter was repressed, but there was no apparent change in A549 and H1975 cells co-transfected with miR-142$5 \mathrm{p}$ mimic and the NFAT5 3'UTR-mut reporter (Figure 5E and F). Also, the expression of NFAT5 mRNA was increased in NSCLC tissues and had a negative correlation with miR-142$5 \mathrm{p}$ (Figure $5 \mathrm{G}$ and $\mathrm{H}$ ). Consistently, the level of NFAT5 protein was elevated in NSCLC tissues and cells (Figure 5I and J). In addition, miR-142-5p mimic reduced the level of NFAT5 protein in NSCLC cells (Figure 5K). Collectively, these results manifested that NFAT5 was a direct target of miR-142-5p.

\section{Circ_0001944 Regulated NFAT5 Expression by Adsorbing miR-142-5p}

Based on the above results, we further analyzed whether circ_0001944 regulated NFAT5 expression by competitively binding to miR-142-5p. There was a positive correlation between NFAT5 and circ_0001944 expression in NSCLC tissues (Figure 6A). Furthermore, the inhibition of circ_0001944 reduced the level of NFAT5 protein in A549 and H1975 cells, but this tendency was reversed after antimiR-142-5p introduction (Figure 6B). These results manifested that circ_0001944 regulated NFAT5 expression by sponging miR-142-5p in NSCLC cells.

\section{Circ_0001944 Accelerated Proliferation, Migration, Invasion, and Increased Glycolysis of NSCLC Cells by Upregulating NFAT5}

To validate whether circ_0001944 regulated proliferation, migration, invasion, and glycolysis of NSCLC cells by regulating NFAT5 expression, we performed rescue experiments. Western blotting was carried out to validate the overexpression efficiency of NFAT5 in 
A

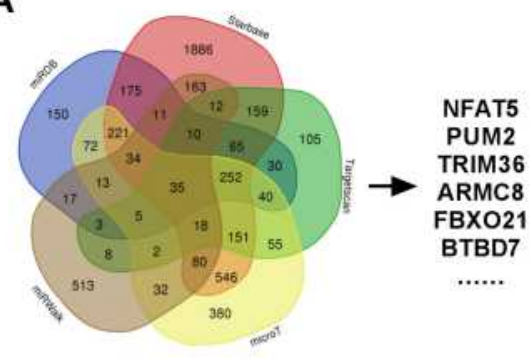

B

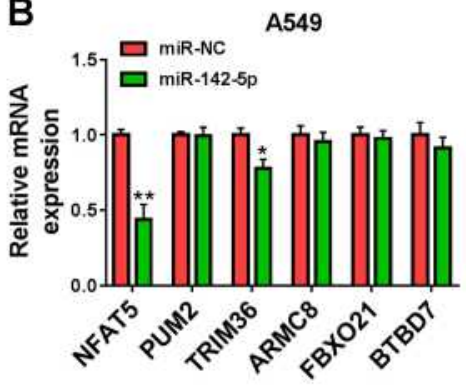

C

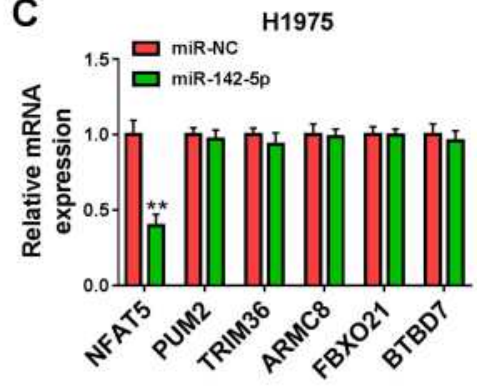

D
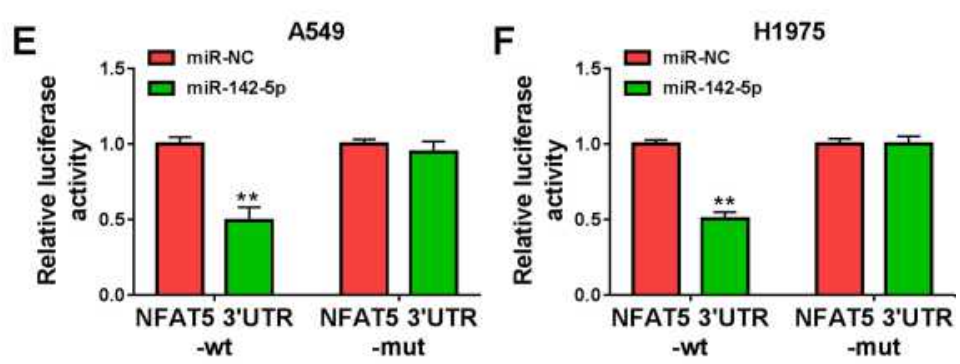

G

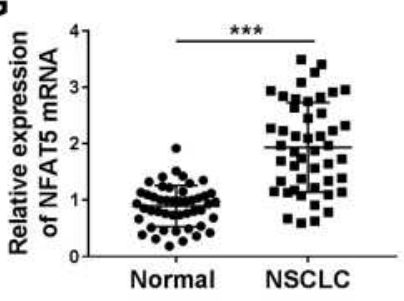

NFAT5 3'UTR wt 5'-GUAAUAUCCAGAGCUACUUUAUA-3' Hsa_miR-142-5p 3'-UCAUCACGAAAGAUGAAAUAC-5' NFAT5 3'UTR mut 5'-UUAAAGAGAGGAGGAUGAAAUAG-3'
H

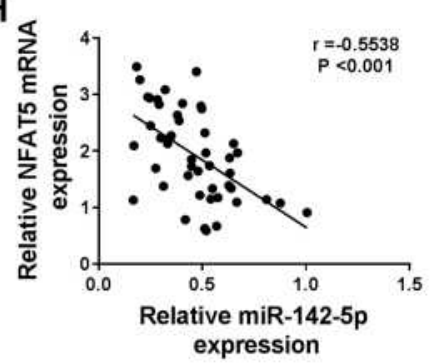

I

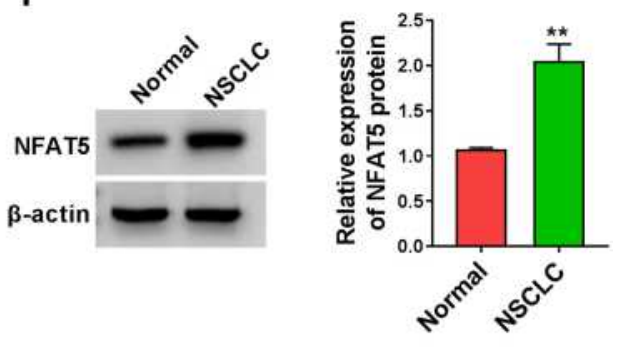

$\mathbf{J}$

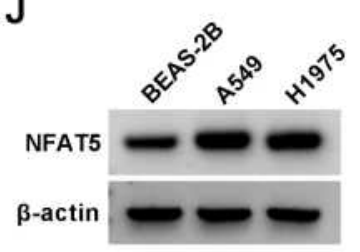

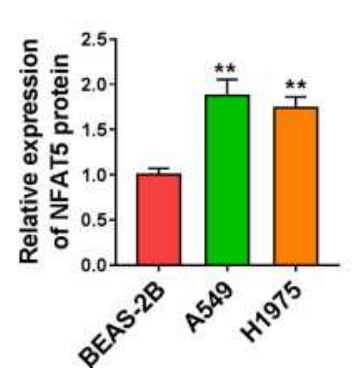

K

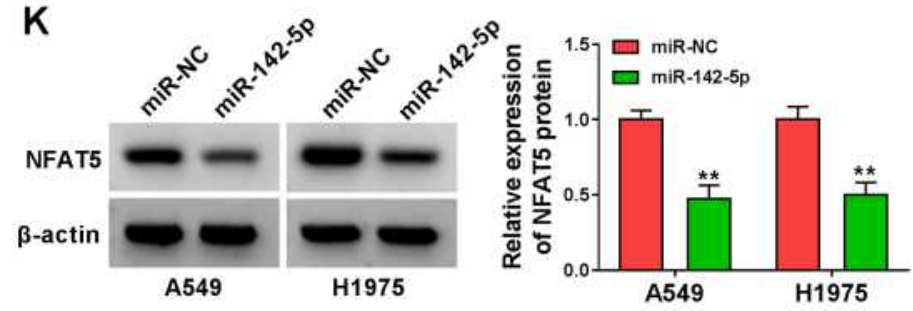

Figure 5 NFAT5 was validated as a direct target of miR-142-5p. (A) The targets of miR-142-5p predicted by bioinformatics (miRDB, Starbase, Targetscan, microT, and miRWalk) were analyzed by Venn. (B and C) Influence of miR-142-5p mimic on expression levels of NFAT5, PUM2, TRIM36, ARMC8, FBXO2I, and BTBD7 in A549 and HI975 cells was assessed by qRT-PCR. (D) Schematic illustration showed the putative binding sites between NFAT5 3'UTR and miR-142-5p. (E and F) The putative binding sites between NFAT5 3'UTR and miR-142-5p were validated by dual-luciferase reporter assay. (G) QRT-PCR analysis of the expression of NFAT5 mRNA in NSCLC tissues. (H) Pearson's correlation analysis revealed the correlation between miR-142-5p and NFAT5 in NSCLC tissues. (I and J) Western blotting revealed the protein level of NFAT5 in NSCLC tissues and cells. (K) Effect of miR-142-5p overexpression on the protein level of NFAT5 was assessed by Western blotting. $* P<0.05$, $* * P<0.01$, and $* * * P<0.001$.

A549 and H1975 cells, as exhibited Figure 7A. Moreover, the forcing expression of NFAT5 offset the inhibitory effect of circ_0001944 knockdown on proliferation, migration, and invasion of A549 and H1975 cells (Figure 7B-F). Also, NFAT5 overexpression reversed the decrease in glucose uptake and lactate production in A549 and H1975 cells mediated by circ_0001944 silencing (Figure 7G and H). In addition, NFAT5 overexpression overturned circ_0001944 inhibition-mediated influence on the protein levels of HK2 and LDHA in A549 and H1975 cells (Figure 7I). These results indicated that circ_0001944 modulated proliferation, migration, invasion, and glycolysis of NSCLC cells through NFAT5. 

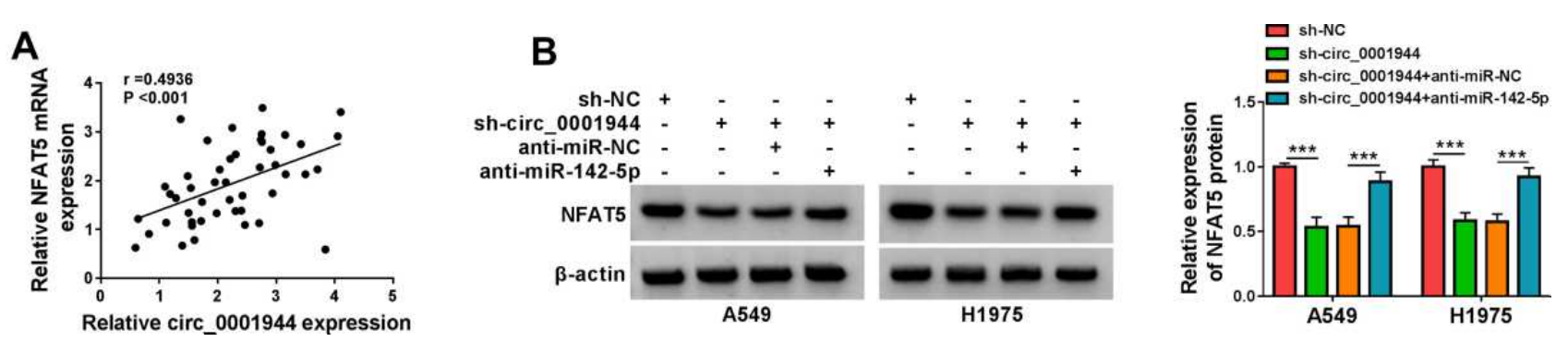

Figure 6 Circ_0001944 regulated NFAT5 expression by adsorbing miR-142-5p. (A) Pearson's correlation analysis presented the correlation between NFAT5 and circ_0001944 in NSCLC tissues. (B) Western blotting detected the protein level of NFAT5 in A549 and HI975 cells transfected with sh-NC, sh-circ_000I944, sh-circ _0001944+anti-miR-NC, or sh-circ_0001944+anti-miR-142-5p. ****P $<0.001$.

A

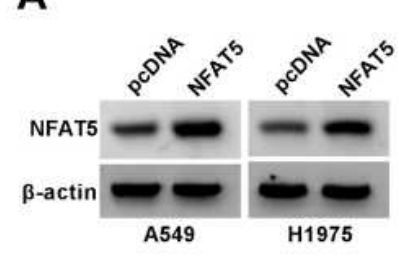

D $\square$ sh-NC+pcDNA

$\square$ sh-circ_0001944+pcDNA

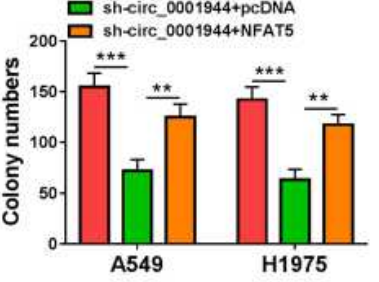

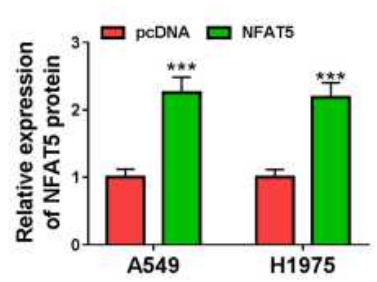

E 口 sh-NC+pcDNA 口 sh-circ_0001944+pcDNA ๖ 200 sh-circ_0001944+NFAT5

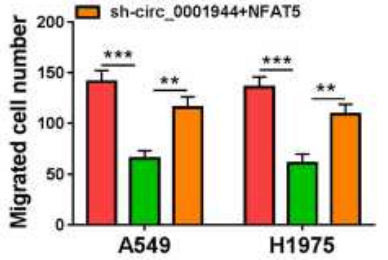

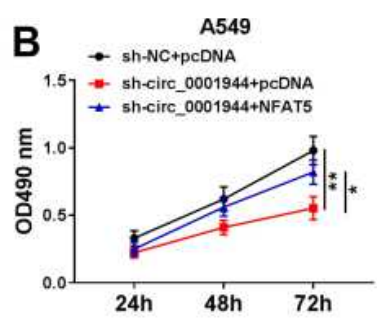

F

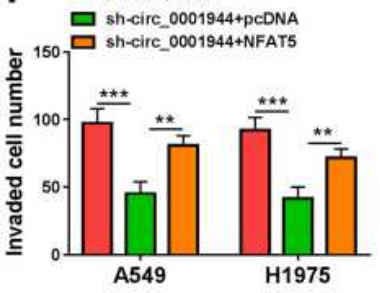

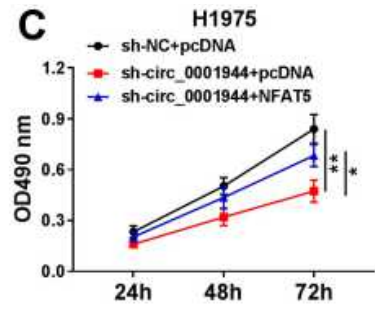

G $\square$ sh-NC+pcDNA

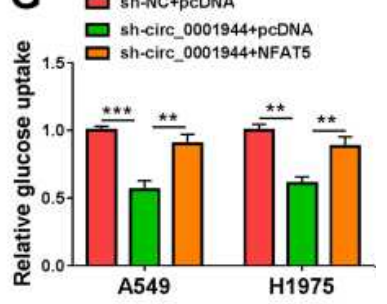

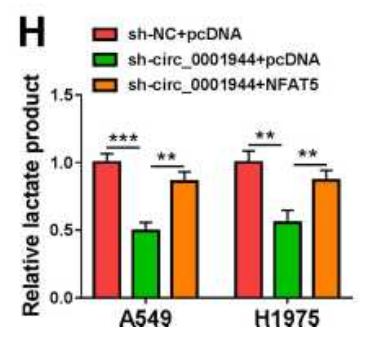
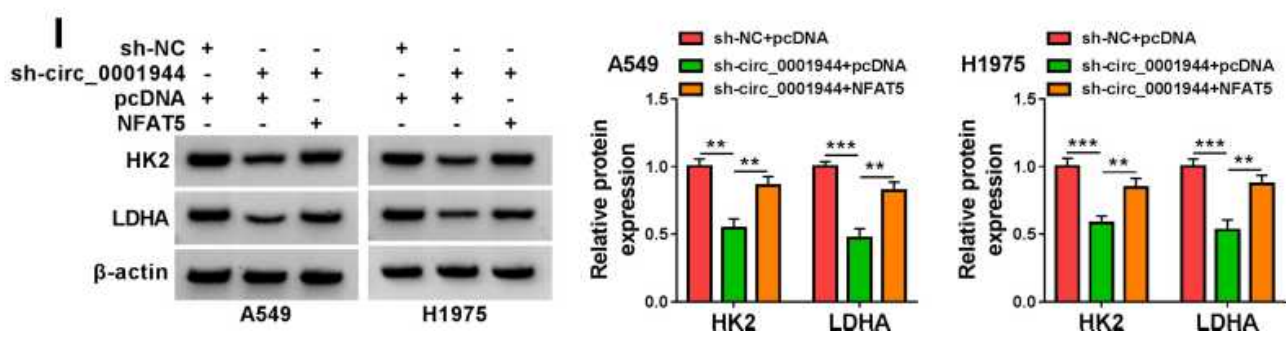

Figure 7 Circ_0001944 promoted proliferation, migration, invasion, and glycolysis of NSCLC cells through NFAT5. (A) After NFAT5 or pcDNA transfection, the level of NFAT5 protein was detected by Western blotting. (B-I) A549 and HI975 cells were transfected with sh-NC+pcDNA, sh-circ_000I944+pcDNA, or sh-circ_000I944 +NFAT5. (B-F) The proliferation, migration, and invasion of A549 and HI 975 cells were determined by MTT, colony formation, and transwell assays. (G and $\mathbf{H}$ ) Analysis of glucose uptake and lactate production in A549 and HI 975 cells using automatic biochemical analyzer. (I) Western blotting assessed protein levels of HK2 and LDHA in A549 and $\mathrm{HI} 975$ cells. $* \mathrm{P}<0.05$, $* * \mathrm{P}<0.0 \mathrm{I}$, and $* * * \mathrm{P}<0.00 \mathrm{I}$.

\section{Circ_0001944 Knockdown Repressed Xenograft Tumor Growth in vivo}

To validate the tumorigenicity of circ_0001944 in NSCLC in vivo, we constructed xenograft mice by injecting with A549 cells carrying sh-circ_0001944 or sh-NC. Compared to the control group, xenograft tumor growth was slower and tumor weight was lower in the sh-circ_0001944 group (Figure 8A and B). Also, the expression levels circ_0001944 and NFAT5 protein were decreased in xenograft tumors in the sh-circ_0001944 group compared with the control group, but miR-142-5p expression was elevated (Figure $8 \mathrm{C}$-E). These results manifested that the silence of circ_0001944 reduced NSCLC growth in vivo. 


\section{A}

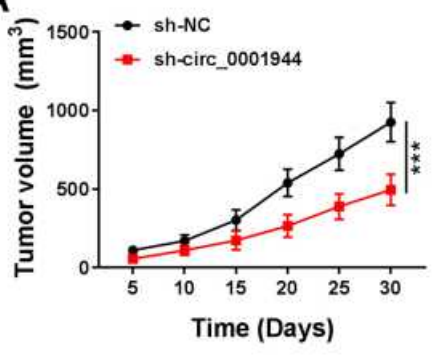

D

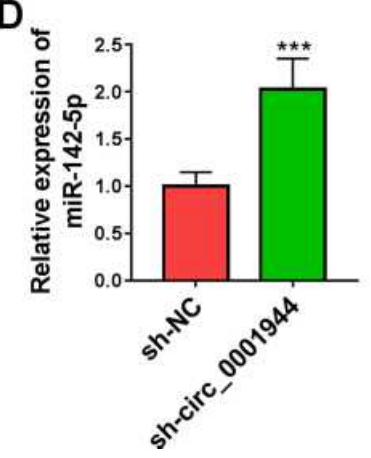

B

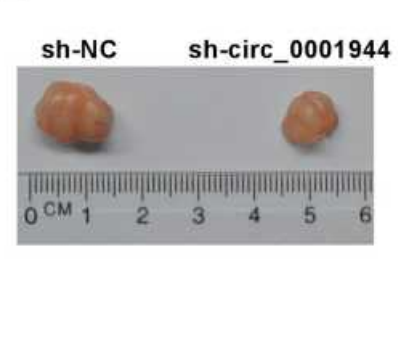

E

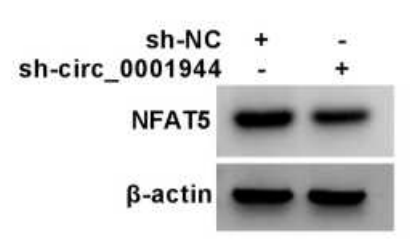

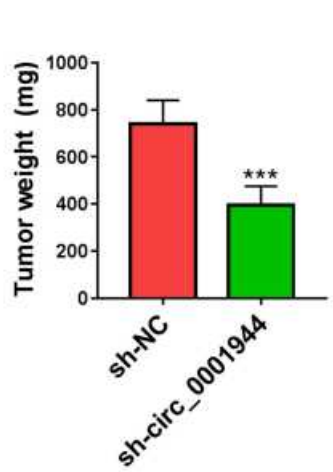

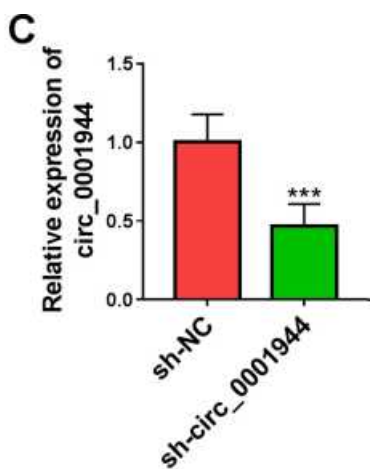

Figure 8 Circ_0001944 silencing curbed xenograft tumor growth in vivo. (A) Tumor growth curves were plotted using xenograft tumor volume data. (B) Tumor weight was assessed after tumor excision. (C and D) Expression tendencies of circ_0001944 and miR-142-5p in xenograft tumors were analyzed by qRT-PCR. (E) The level of NFAT5 protein in xenograft tumors was detected by Western blotting. $* * * P<0.001$.

\section{Discussion}

A large number of circRNAs have been identified to be abnormally expressed in NSCLC through RNA sequencing. ${ }^{14}$ However, the functions and regulatory mechanisms of many abnormally expressed circRNAs in NSCLC are still unclear. In this study, we verified that circ_0001944 accelerated cell glycolysis and malignancy by regulating the miR-142-5p/NFAT5 pathway in NSCLC.

Glycolysis not only provides energy for cancer cells but also produces important metabolic intermediates, which exert crucial roles in maintaining cell proliferation, migration, and invasion. ${ }^{16,17} \mathrm{HK} 2$ is a speed-limiting enzyme in the glycolysis process. ${ }^{18}$ LDHA, an important HIF-1 $\alpha$ target, can catalyze the reduction of pyruvate to lactate and maintain cell survival under hypoxic conditions. ${ }^{19}$ Our data verified that circ_0001944 was upregulated in NSCLC, and NSCLC patients with high circ_0001944 expression had a worse prognosis, indicating that circ_0001944 might be an unfavorable biomarker for NSCLC patients. Moreover, circ_0001944 silencing decreased xenograft tumor growth in vivo and constrained proliferation, metastasis, and invasion of NSCLC cells in vitro, suggesting that circ_0001944 played a promoting effect on tumor progression in NSCLC cells. Also, circ_0001944 knockdown reduced glucose uptake, lactate production, and protein levels of HK2 and LDHA in NSCLC cells, manifesting that circ_0001944 facilitated cell glycolysis in NSCLC. Previous studies had proved that circ_0001944 was upregulated in breast cancer brain metastasis cells ${ }^{20}$ and NSCLC tissues. ${ }^{14}$ In addition, high circ_0001944 expression facilitated bladder cancer advancement by upregulating PROK2 via sponging miR$548 .^{13}$ Therefore, we concluded that circ_0001944 played a promoting effect on cell tumor growth and glycolysis in NSCLC.

Based on circRNAs that can function as a sponge for miRs, we verified circ_0001944 as a sponge for miR-1425 p. MiR-142 could be used as a prognostic indicator for patients with solid tumors. ${ }^{21}$ It had been proved that miR142-5p participated in the development of many cancers. Previous studies had reported that miR-142-5p repressed the advancement of gastric cancer, breast cancer, and cervical cancer by, respectively, targeting CYR61, SORBS1, and PIK3AP1. ${ }^{22-24}$ However, miR-142-5p played a carcinogenic role in renal cell cancer and colorectal cancer. ${ }^{25,26}$ In addition, miR-142-5p exerted a tumorrepressive role in lung cancer. Jiang et al revealed that 
IncRNA XIST accelerated the tumorigenicity of NSCLC cells through adsorbing miR-142-5p and upregulating PAX6. $^{27}$ Also, circRNA 0014130 sponged miR-142-5p to increase IGF-1 expression, thereby curbing cell apoptosis and facilitating cell proliferation in NSCLC. ${ }^{28}$ Herein, miR-142-5p was lowly expressed in NSCLC. The silence of miR-142-5p overturned the inhibitory influence of circ_0001944 knockdown on proliferation, migration, invasion, and glycolysis of NSCLC cells. Accordingly, these data indicated that circ_0001944 promoted tumor growth and glycolysis of NSCLC cells through adsorbing miR-142-5p.

The NFAT family of transcription factors is involved in cell cycle, angiogenesis, and metastasis. ${ }^{30}$ NFAT proteins also take part in metabolic signaling and effector functions. $^{29}$ NFAT5, a member of the Rel transcription factor family, is involved in the pathogenesis of diverse cancers. It was reported that NFAT5 acted as an oncogene in renal cancer, breast cancer, pancreatic cancer, and glioblastoma. ${ }^{31-34}$ Furthermore, NFAT5 was identified as an independent risk factor for DFS of NSCLC patients. ${ }^{35}$ Meng et al indicated that miR-194 curbed high glucoseinduced malignant behaviors of NSCLC cells by targeting NFAT5. ${ }^{36}$ Herein, NFAT5 was verified as a target of miR142-5p. Notably, circ_0001944 regulated NFAT5 expression by adsorbing miR-142-5p. Moreover, NFAT5 overexpression offset the suppressive impact of circ_0001944 downregulation on proliferation, migration, invasion, and glycolysis of NSCLC cells. Therefore, we inferred that circ_0001944 elevated NFAT5 expression by adsorbing miR-142-5p, resulting in facilitating tumor growth and glycolysis of NSCLC cells.

In conclusion, circ_0001944 exerted a carcinogenic role in NSCLC. Furthermore, circ_0001944 accelerated proliferation, migration, invasion, and glycolysis of NSCLC cells by elevating NFAT5 expression through adsorbing miR-142-5p. The research offered a new mechanism responsible for NSCLC advancement and manifested that circ_0001944 might be an underlying target for NSCLC treatment.

\section{Funding}

There is no funding to report.

\section{Disclosure}

The authors reported no conflicts of interest for this work.

\section{References}

1. Alexander M, Kim SY, Cheng H. Update 2020: management of non-small cell lung cancer. Lung. 2020;198(6):897-907.

2. Jin J, Robeson H, Fagan P, Orloff MS. Association of PARP1-specific polymorphisms and haplotypes with non-small cell lung cancer subtypes. PLoS One. 2020;15(12):e0243509.

3. Heigener DF, Reck M. Lung cancer in 2017: giant steps and stumbling blocks. Nat Rev Clin Oncol. 2018;15(2):71-72.

4. Miller KD, Nogueira L, Mariotto AB, et al. Cancer treatment and survivorship statistics, 2019. CA Cancer J Clin. 2019;69(5):363-385.

5. Hanahan D, Weinberg RA. Hallmarks of cancer: the next generation. Cell. 2011;144(5):646-674.

6. Shankaraiah RC, Veronese A, Sabbioni S, Negrini M. Non-coding RNAs in the reprogramming of glucose metabolism in cancer. Cancer Lett. 2018;419:167-174.

7. Hsiao KY, Sun HS, Tsai SJ. Circular RNA - New member of noncoding RNA with novel functions. Exp Biol Med (Maywood). 2017;242(11):1136-1141.

8. Qu S, Yang X, Li X, et al. Circular RNA: a new star of noncoding RNAs. Cancer Lett. 2015;365(2):141-148.

9. Liang ZZ, Guo C, Zou MM, Meng P, Zhang TT. circRNA-miRNAmRNA regulatory network in human lung cancer: an update. Cancer Cell Int. 2020;20:173.

10. Verduci L, Strano S, Yarden Y, Blandino G. The circRNA-microRNA code: emerging implications for cancer diagnosis and treatment. $\mathrm{Mol}$ Oncol. 2019;13(4):669-680.

11. Wang H, Sun G, Xu P, et al. Circular RNA TMEM87A promotes cell proliferation and metastasis of gastric cancer by elevating ULK1 via sponging miR-142-5p. J Gastroenterol. 2021;56(2):125-138.

12. Mao Y, He JX, Zhu M, Dong YQ, He JX. Circ0001320 inhibits lung cancer cell growth and invasion by regulating TNFAIP1 and TPM1 expression through sponging miR-558. Hum Cell. 2021;34 (2):468-477.

13. Jin M, Lu S, Wu Y, et al. Hsa_circ_0001944 promotes the growth and metastasis in bladder cancer cells by acting as a competitive endogenous RNA for miR-548. J Exp Clin Cancer Res. 2020;39 (1): 186 .

14. Hang D, Zhou J, Qin N, et al. A novel plasma circular RNA circFARSA is a potential biomarker for non-small cell lung cancer. Cancer Med. 2018;7(6):2783-2791.

15. Zhou L, Li M, Yu X, Gao F, Li W. Repression of Hexokinases II-mediated glycolysis contributes to piperlongumine-induced tumor suppression in non-small cell lung cancer cells. Int $J$ Biol Sci. 2019;15(4):826-837.

16. Abdel-Wahab AF, Mahmoud W, Al-Harizy RM. Targeting glucose metabolism to suppress cancer progression: prospective of anti-glycolytic cancer therapy. Pharmacol Res. 2019;150:104511.

17. Pezzuto A, D'Ascanio M, Ricci A, Pagliuca A, Carico E. Expression and role of p16 and GLUT1 in malignant diseases and lung cancer: a review. Thorac Cancer. 2020;11(11):3060-3070.

18. Garcia SN, Guedes RC, Marques MM. Unlocking the Potential of HK2 in cancer metabolism and therapeutics. Curr Med Chem. 2019;26(41):7285-7322.

19. Pathria G, Scott DA, Feng Y, et al. Targeting the Warburg effect via LDHA inhibition engages ATF4 signaling for cancer cell survival. EMBO J. 2018;37(20):E99735.

20. Fu B, Zhang A, Li M, et al. Circular RNA profile of breast cancer brain metastasis: identification of potential biomarkers and therapeutic targets. Epigenomics. 2018;10(12):1619-1630.

21. Liu R, Zheng S, Yu K, et al. Prognostic value of miR-142 in solid tumors: a meta-analysis. Biosci Rep. 2021;41(2):BSR20204043.

22. Yan J, Yang B, Lin S, Xing R, Lu Y. Downregulation of miR-142-5p promotes tumor metastasis through directly regulating CYR61 expression in gastric cancer. Gastric Cancer. 2019;22(2):302-313. 
23. Yu W, Li D, Zhang Y, et al. MiR-142-5p acts as a significant regulator through promoting proliferation, invasion, and migration in breast cancer modulated by targeting SORBS1. Technol Cancer Res Treat. 2019;18:1533033819892264.

24. Guo J, Tang T, Li J, et al. Overexpression of miR-142-5p suppresses the progression of cervical cancer through targeting PIK3AP1 expression. Mol Cell Biol. 2020.

25. Liu L, Liu S, Duan Q, et al. MicroRNA-142-5p promotes cell growth and migration in renal cell carcinoma by targeting BTG3. Am J Transl Res. 2017;9(5):2394-2402.

26. Liu S, Xiao Z, Ai F, et al. miR-142-5p promotes development of colorectal cancer through targeting SDHB and facilitating generation of aerobic glycolysis. Biomed Pharmacother. 2017;92:1119-1127.

27. Jiang Q, Xing W, Cheng J, Yu Y. Knockdown of lncRNA XIST suppresses cell tumorigenicity in human non-small cell lung cancer by regulating mir-142-5p/PAX6 axis. OncoTargets Ther. 2020;13:4919-4929.

28. Wang M, Shi J, Jiang H, Xu K, Huang Z. Circ_0014130 participates in the proliferation and apoptosis of nonsmall cell lung cancer cells via the miR-142-5p/IGF-1 axis. Cancer Biother Radiopharm. 2020;35(3):233-240

29. Prasad M, Brzostek J, Gautam N, Balyan R, Rybakin V, Gascoigne NRJ. Themis regulates metabolic signaling and effector functions in CD4 T cells by controlling NFAT nuclear translocation. Cell Mol Immunol. 2020.
30. Mognol GP, Carneiro FRG, Robbs BK, Faget DV, Viola JPB. Cell cycle and apoptosis regulation by NFAT transcription factors: new roles for an old player. Cell Death Dis. 2016;7:e2199.

31. Küper C, Beck FX, Neuhofer W. NFAT5-mediated expression of S100A4 contributes to proliferation and migration of renal carcinoma cells. Front Physiol. 2014;5:293.

32. Matsuda M, Katakura Y, Hirata M, et al. Nuclear factor of activated T cells 5 maintained by Hotair suppression of miR-568 upregulates S100 calcium binding protein A4 to promote breast cancer metastasis. Lab Invest. 2014;16(5):454.

33. Jiang Y, He R, Jiang Y, et al. Transcription factor NFAT5 contributes to the glycolytic phenotype rewiring and pancreatic cancer progression via transcription of PGK1. Cell Death Dis. 2019;10(12):948.

34. Zhang S, Liao K, Miao Z, et al. CircFOXO3 promotes glioblastoma progression by acting as a competing endogenous RNA for NFAT5. Cell Death Dis. 2019;21(10):1284-1296.

35. Cho HJ, Yun HJ, Yang HC, et al. Prognostic significance of nuclear factor of activated T-cells 5 expression in non-small cell lung cancer patients who underwent surgical resection. $J$ Surg Res. 2018;226:40-47.

36. Meng X, Li Z, Zhou S, Xiao S, Yu P. miR-194 suppresses high glucose-induced non-small cell lung cancer cell progression by targeting NFAT5. Thorac Cancer. 2019;10(5):1051-1059.

\section{Publish your work in this journal}

Cancer Management and Research is an international, peer-reviewed open access journal focusing on cancer research and the optimal use of preventative and integrated treatment interventions to achieve improved outcomes, enhanced survival and quality of life for the cancer patient.
The manuscript management system is completely online and includes a very quick and fair peer-review system, which is all easy to use. Visit http://www.dovepress.com/testimonials.php to read real quotes from published authors. 